Proc. Indian Acad. Sci. (Math. Sci.), Vol. 107, No. 1, February 1997, pp. 89-93.

(C) Printed in India

\title{
On the incoming water waves against a vertical cliff
}

\author{
A CHAKRABARTI and T SAHOO \\ Department of Mathematics, Indian Institute of Science, Bangalore 560012, India
}

MS received 6 June 1996; revised 10 September 1996

\begin{abstract}
The problems of obliquely incident surface water waves against a vertical cliff have been handled in both the cases of water of infinite as well as finite depth by straightforward uses of appropriate Havelock-type expansion theorems. The logarithmic singularity along the shore-line has been incorporated in a direct manner, by suitably representing the Dirac's delta function.
\end{abstract}

Keywords. Water wave; logarithmic singularity; Dirac's delta function.

\section{Introduction}

The problem of incoming incident surface water waves normally incident against a vertical cliff, in the linearized theory was first investigated by Stoker [6] (see also Stoker [7]) by complex variable method. Assuming that there is no reflection of these waves, a source or sink type behaviour at the shore line was introduced, to account for the fact that the wave amplitude remains finite at the origin. The problem of incoming surface water waves normally incident on a vertical cliff was reinvestigated by Mandal and Kundu [5] in the case of water of infinite depth. The problem of obliquely incident water waves against a vertical cliff was investigated by Chakrabarti [1] in the case of water of infinite depth by using the commutativity property of the differential operators appearing in the boundary conditions and the case of normally incident surface water wave was handled as a particular case.

In this note, we have employed a simple and straightforward method, by using the Havelock's expansion theorem to solve the problem of obliquely incident surface water waves by a vertical cliff in both the cases of water of infinite as well as finite depths. The method of solution of the boundary value problems under consideration is based on the exploitation of the logarithmic singularity of the velocity potential at the corner point, where the water surface meets the wall in a rather natural manner, while applying the expansion theorem of Havelock. The solutions in the case of the problems of normally incident surface water waves are reproduced as particular cases.

\section{Mathematical formulation}

The problems under consideration are three dimensional in nature and these problems will be studied under the assumption that the incoming waves are incident against the vertical cliff with an angle $\alpha(0<\alpha<\pi / 2)$. Assuming that the cliff occupies the position $x=0,0<y<\infty$ in the case of water of infinite depth $(x=0,0<y<h$ in the case of water of finite depth) in the Cartesian co-ordinate system and the fluid occupies the region $x>0, y>0$ in the case of water of infinite depth, whereas, in case of water of finite depth, the fluid occupies the region $x>0,0<y<h$ where $y=h$ is the bottom surface in the case of water of finite depth. If we also assume that the 
fluid is inviscid, incompressible, the motion irrotational and simple harmonic in time, we will have the existence of the velocity potential $\Phi(x, y, z, t)$ such that $\Phi(x, y, z, t)=\operatorname{Re}\left(\phi(x, y) \mathrm{e}^{-i \omega t+i K z \sin \alpha}\right)$ in the case of water of infinite depth (K is replaced by $k_{0}$ in the case of water of finite depth, where $k_{0}$ is the real and positive root of the transcendental equation $\alpha \tan h \alpha h=K$ in $\alpha$ ) (see Jervis and Taylor [4], Faulkner [3]). Then under the assumption of the linearized theory, the problem of obliquely incident surface water waves against a vertical cliff can be investigated by way of determining the potential $\phi(x, y)$ [after dropping the factor $\mathrm{e}^{-i \omega t+i K z \sin \alpha}$ from the whole mathematical formulation, (see Chakrabarti [1])] which satisfies the boundary value problem as given by

(i) the partial differential equation

$$
\frac{\partial^{2} \phi}{\partial x^{2}}+\frac{\partial^{2} \phi}{\partial y^{2}}-v^{2} \phi=0, \text { in the fluid region, }
$$

(ii) the linearized free surface condition

$$
\frac{\partial \phi}{\partial y}+K \phi=0, \quad \text { on } \quad y=0 \quad K>0
$$

where $K=\omega^{2} / g, g$ the acceleration due to gravity,

(iii) the condition on the vertical wall

$$
\frac{\partial \phi}{\partial x}=0, \text { on } x=.0, \text { for } y>0
$$

(iv) the condition at the corner point

$$
\phi \rightarrow \ln \sqrt{x^{2}+y^{2}} \text { as } \sqrt{x^{2}+y^{2}} \rightarrow 0,
$$

(v) the infinity conditions

$$
\phi(x, y) \rightarrow \mathrm{e}^{-K y-i \mu x} \text { as } x \rightarrow \infty,
$$

in the case of water of infinite depth. Whereas in the case of water of finite depth,

and

$$
\phi(x, y) \rightarrow \frac{\mathrm{e}^{-i \mu x} \cosh k_{0}(h-y)}{\cosh k_{0} h} \text { as } x \rightarrow \infty,
$$

(vi) the bottom conditions

$$
\phi,|\nabla \phi| \rightarrow 0 \quad \text { as } y \rightarrow \infty,
$$

in the case of water of infinite depth. Whereas, in the case of water of finite depth

$$
\frac{\partial \phi}{\partial y}=0 \quad \text { on } \quad y=h \text {. }
$$

In (2.1) and (2.5), $v=K \sin \alpha$ and $\mu=K \cos \alpha$ in the case of water of infinite depth, and $v=k_{0} \sin \alpha, \mu=k_{0} \cos \alpha$ in the case of water of finite depth, $k_{0}$ being the same as defined earlier. 


\subsection{The method of solution for water of infinite depth}

From the condition (2.4), we have

$$
\frac{\partial \phi}{\partial x} \approx \frac{x}{x^{2}+y^{2}} \text { for } \sqrt{x^{2}+y^{2}} \rightarrow 0 .
$$

But we have (see Friedman [2])

$$
\lim _{x \rightarrow 0} \frac{x}{x^{2}+y^{2}}=\pi \delta(y) \text { for all } y,
$$

where $\delta(y)$ is the Dirac's delta function and the limit in (2.10) is understood in the sense of distribution.

Hence

$$
\lim _{x \rightarrow 0} \frac{\partial \phi}{\partial x} \approx \pi \delta(y) \text { for all } y
$$

which gives, after using the condition (2.3), that

$$
\frac{\partial \phi}{\partial x}=C \delta(y) \text { on } x=0, y \geqslant 0,
$$

where $C$ is a suitable constant, which can be chosen as explained below.

The solution for water of infinite depth can be expressed as

$$
\phi(x, y)=\mathrm{e}^{-K y-i \mu x}+\frac{2}{\pi} \int_{0}^{\infty} \frac{A(\xi)(\xi \cos \xi y-K \sin \xi y) \mathrm{e}^{-\sqrt{\xi^{2}+v^{2}} \cdot x} \mathrm{~d} \xi}{\xi^{2}+K^{2}},
$$

where $A(\xi)$ is an unknown function to be determined.

Using the condition (2.12) in the expression (2.13), we obtain

$$
-i \mu \mathrm{e}^{-K y}-\frac{2}{\pi} \int_{0}^{\infty} \frac{\sqrt{\xi^{2}+v^{2}} A(\xi)(\xi \cos \xi y-K \sin \xi y) \mathrm{d} \xi}{\xi^{2}+K^{2}}=C \delta(y), \quad 0 \leqslant y \leqslant \infty .
$$

By Havelock's expansion theorem, we have

$$
C=\frac{-i u}{2 K} \text { and } \xi A(\xi)=-\frac{C \xi}{\sqrt{\xi^{2}+v^{2}}}
$$

which gives

$$
A(\xi)=\frac{i \xi \cos \alpha}{2 \sqrt{\xi^{2}+v^{2}}} .
$$

Substituting for $A(\xi)$ from (2.16) in (2.13), we obtain

$$
\phi(x, y)=\mathrm{e}^{-K y-i \mu x}+\frac{i \cos \alpha}{\pi} \int_{0}^{\infty} \frac{(\xi \cos \xi y-K \sin \xi y) \mathrm{e}^{-\sqrt{\xi^{2}+v^{2}} \cdot x} \mathrm{~d} \xi}{\sqrt{\xi+v^{2}}\left(\xi^{2}+K^{2}\right)}
$$

which is the full solution in this case, agreeing with the one obtained by Chakrabarti[1] earlier. In particular, when $\alpha=0$, the velocity potential $\phi(x, y)$ for water of infinite depth agrees with the result obtained by Mandal and Kundu [5]. 


\subsection{Solution for water of finite depth}

Here we discuss the solution procedure in the case when the water is of finite depth. The solution is of the form as given by

$$
\phi(x, y)=\frac{\mathrm{e}^{-i \mu x} \cosh k_{0}(h-y)}{\cosh k_{0} h}+\sum_{n=1}^{\infty} A_{n} \mathrm{e}^{-\sqrt{k_{n}^{2}+v^{2} \cdot x}} \cos k_{n}(h-y),
$$

where $A_{n}$ 's are the unknowns to be determined and $k_{n}, n=1,2, \ldots, n$ are the roots of the transcendental equation $K+\alpha \tan \alpha h=0$ in $\alpha$. As in the previous case, we have from the conditions (2.3) and (2.4)

$$
\frac{\partial \phi}{\partial x}=C^{\prime} \delta(y), \quad 0 \leqslant y<h,
$$

where $C^{\prime}$ is a suitable constant to be determined.

The expression (2.18) along with the condition (2.19) give rise to the relation

$$
\frac{-i \mu \cosh k_{0}(h-y)}{\cosh k_{0} h}-\sum_{n=1}^{\infty} A_{n} \sqrt{k_{n}^{2}+v^{2}} \cos k_{n}(h-y)=C^{\prime} \delta(y), \quad 0 \leqslant y<h .
$$

By using the extension of Havelock's expansion theorem as required for problems involving fluids of finite depth (see Ursell [8]), we obtain from (2.20) that

which gives

$$
C^{\prime}=-\frac{i \mu}{4 k_{0}} \frac{2 k_{0} h+\sinh 2 k_{0} h}{\cosh ^{2} k_{0} h} \quad \text { and } \quad A_{n}=\frac{-4 C^{\prime} k_{n} \cos k_{n} h}{\left(2 k_{0} h+\sin 2 k_{n} h\right) \sqrt{k_{n}^{2}+v^{2}}}
$$

$$
A_{n}=\frac{2 i k_{n} \cos \alpha\left(2 k_{0} h+\sin 2 k_{0} h\right) \cos k_{n} h}{\sqrt{k_{n}^{2}+v^{2}}\left(1+\cosh 2 k_{0} h\right)\left(2 k_{n} h+\sin 2 k_{n} h\right)} .
$$

Thus the final solution is obtained by substituting for $A_{n}$ in the expression (2.18) for $\phi(x, y)$. In particular, when $\alpha=0$ the solution for the case of normally incident wave can be obtained.

\section{Conclusion}

The problems of obliquely incident water waves by a vertical wall have been investigated in a very simple and systematic manner, in both the cases of water of infinite as well as finite depths, by suitable application of Havelock's expansion theorem to the condition on the vertical cliff and the singularity behaviour at the edge.

\section{Acknowledgements}

The authors acknowledge the referee for his suggestions and comments on the paper. TS acknowledges CSIR, New Delhi for the financial support as a research associate of Indian Institute of Science.

\section{References}

[1] Chakrabarti A, Obliquely incident water waves against a vertical cliff, Appl. Math. Lett. 5 (1992) 13-17

[2] Friedman B, Principles and Techniques of Applied Mathematics (1956) (New York: John Wiley and Sons Inc.) 
[3] Faulkner T R, The diffraction of an obliquely incident surface water wave by a vertical barrier of finite depth, Proc. Cambridge Philos. Soc. 62 (1966) 829-838

[4] Jervis J R and Taylor B S, The Scattering of surface water waves by a vertical plane barrier, Proc. Cambridge Philos. Soc. 66 (1969) 417-422

[5] Mandal B N and Kundu P K, Incoming water waves against a vertical cliff, Appl. Math. Lett. 3 (1990) 33-36

[6] Stoker J J, Water Waves (1957) (New York: Interscience)

[7] Stoker J J, Surface waves in water of variable depth, Quart. Appl. Math. 5 (1947) 1-54

[8] Ursell F, The effect of a fixed vertical barrier on surface waves in deep water, Proc. Cambridge. Philos. Soc. 43 (1947) 374-382 